\title{
Podocyturia predicts pre-eclampsia
}

New data suggest that detection of podocyturia (that is, the shedding of viable podocytes in urine) might serve as an early diagnostic test for pre-eclampsia prior to the development of overt proteinuria and hypertension. Vesna Garovic and colleagues previously showed that podocyturia is present at delivery in women with preeclampsia. Now they report the presence of podocyturia earlier in pregnancy in women subsequently diagnosed with the disease.

To investigate whether podocyturia can be detected early in pregnancy and could serve as an early marker for the development of proteinuria and preeclampsia, the researchers analysed blood and urine samples collected from women at the end of the second trimester of pregnancy and within $24 \mathrm{~h}$ of their deliveries. They found that podocyturia (identified by culturing urinary sediments for $24 \mathrm{~h}$ and then staining for podocin) was present at the end of the second trimester and shortly after delivery in all of the 15 women who developed pre-eclampsia and in none of the 44 women who remained normotensive throughout pregnancy. Of the 15 women who developed gestational hypertension, none had podocyturia at the end of the second trimester and one had podocyturia shortly after delivery. By contrast, considerable overlap was observed between the levels of the angiogenic factors soluble fms-like tyrosine kinase receptor-1, placental growth factor and endoglin in women who developed pre-eclampsia or gestational hypertension and in those who remained normotensive, both in blood samples collected at the end of the second trimester and in those collected within $24 \mathrm{~h}$ of delivery.

The researchers' statistical analysis confirmed that podocyturia at the end of the second trimester was a more sensitive and more specific marker of a subsequent diagnosis of pre-eclampsia than was the level of any of the angiogenic factors or their combinations. "Our data suggest that screening for podocyturia at the end of the second trimester of pregnancy may enable the accurate identification of women at risk of pre-eclampsia," says Garovic.
After adjustment for urine creatinine concentration and time of gestation, the researchers found a significant positive association between urinary podocyte counts and

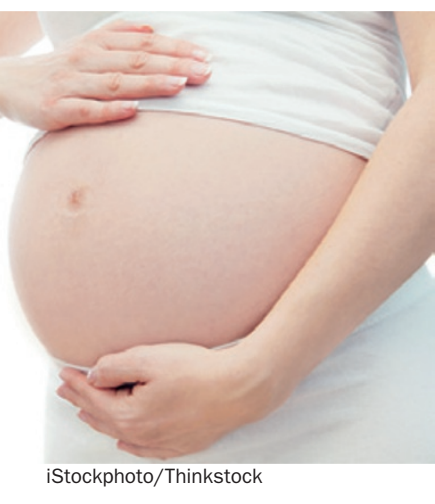
levels of proteinuria in women with pre-eclampsia. "We postulate that loss of viable podocytes during pregnancy in these women might trigger glomerular destabilization, leading to ongoing podocyte loss and ultimately proteinuria," explains Garovic. "This mechanism might underlie the association between preeclampsia and future proteinuria, chronic kidney disease and end-stage renal disease."

Ellen F. Carney

Original article Craici, I. M. et al. Podocyturia predates proteinuria and clinical features of preeclampsia: longitudinal prospective study. Hypertension doi:10.1161/ HYPERTENSIONAHA.113.01115 